Daniel T.L. Shek*, Jianqiang Liang and Li Lin

\title{
Socio-demographic and family predictors of moral competence in Chinese adolescents in Hong Kong: a six-wave longitudinal study
}

DOI 10.1515/ijdhd-2017-5010

Received January 12, 2016; accepted January 24, 2016; previously published online June 7, 2016

Abstract: This six-wave longitudinal study in Project P.A.T.H.S. examined the growth trajectories and predictors of moral competence in Chinese adolescents in Hong Kong. Linear mixed models utilizing individual growth curves were used to analyze the initial status and rate of change of moral competence with reference to gender, economic disadvantage, family intactness, family functioning and parent-child subsystem quality. Results showed that adolescent moral competence increased across 6 years. Female adolescents had higher initial levels of moral competence than that of male adolescents. Adolescents from well-functioning families had higher initial levels of moral competence but slower growth of moral competence than did those from families with lower family functioning. Adolescents with higher mother-child subsystem quality had higher initial levels of moral competence but slower growth of moral competence than did those with relatively lower mother-child subsystem quality. Finally, adolescents with higher father-child subsystem quality had higher initial levels of moral competence than those with relatively lower father-child subsystem quality.

Keywords: Chinese adolescents; family predictors; longitudinal research; moral competence; positive youth development.

\footnotetext{
*Corresponding author: Professor Daniel T.L. Shek, PhD, FHKPS, SBS, JP, Associate Vice President and Chair Professor, Department of Applied Social Sciences, The Hong Kong Polytechnic University, Hunghom, Hong Kong, P.R. China, E-mail: daniel.shek@polyu.edu.hk; Centre for Innovative Programmes for Adolescents and Families, The Hong Kong Polytechnic University, Hong Kong, P.R. China; School of Social Development, East China Normal University, Shanghai, P.R. China; Kiang Wu Nursing College of Macau, Macau, P.R. China; and Division of Adolescent Medicine, Department of Pediatrics, Kentucky Children's Hospital, University of Kentucky College of Medicine, Lexington, KY, USA

Jianqiang Liang and Li Lin: Department of Applied Social Sciences, The Hong Kong Polytechnic University, Hong Kong, P.R. China
}

\section{Introduction}

Moral competence is an important indicator of holistic development in adolescence. Catalano et al. stated that "moral competence is youth's ability to assess and respond to the ethical, affective, or social-justice dimensions of a situation" [1]. Moral competence is also embedded in the virtues of human development [2] which refers to "the knowledge, ability, and motivation to pursue and to do good effectively" [2]. Moral competence is considered as one of the five essential psychosocial competencies in positive youth development, together with social, emotional, cognitive, and behavioral competencies [1]. In this study, "moral competence" refers to "the affective orientation to perform altruistic behaviors towards others and the ability to judge moral issues logically, consistently, and at an advanced level of development" [3]. The study examined the developmental trajectory (from Grade 7 to Grade 12) of moral competence in the individual, familial and social contexts of Chinese adolescents in Hong Kong. This concept was measured by the self-perception of adolescents' moral orientation and moral judgment in this study.

As a key part of the socialization process, adolescent moral development is influenced by personal and family factors [4, 5]. Eisenberg et al. [4] conducted a series of longitudinal studies on adolescent development focusing on prosocial functioning and moral reasoning, but the studies were mainly confined to different types of moral reasoning that adolescents used in a particular moral dilemma situation. Besides, few studies examined adolescents' perceptions of their own moral competence using longitudinal data. Furthermore, moral competence may be associated with other factors, such as biological, psychological, social, cultural, ecological correlates across time [6]. However, few empirical studies have examined the status and patterns of change of adolescent moral competence with reference to different individual, family and social contextual factors.

The concept of moral competence has been commonly incorporated in some youth enhancement programs. In Hong Kong, moral competence is one of the 15 key positive 
youth development constructs which is assessed by the Chinese positive youth development scale [7-9]. Previous research of the project demonstrated that moral competence, together with other positive youth development constructs, had positive impacts on adolescent development, especially in their prosocial norms and behaviors $[10,11]$.

Research of adolescent moral development in the West showed that morality of adolescents developed across time $[4,5]$. In their longitudinal studies on prosocial disposition from mid-adolescent to early adulthood, Eisenberg et al. [4] found that perspective taking and moral reasoning increased in maturity. On the other hand, empirical studies on moral development in the Chinese societies also showed positive development of moral competence in adolescent years [12-14]. In particular, positive parent-child and sibling relationships promoted adolescent moral development - adolescents gradually acquired and developed a mature understanding of moral codes (e.g. showing filial piety to one's parents) over time $[13,14]$.

Regarding gender and moral competence, there are no conclusive findings in the scientific literature. Some scholars provided research evidence to show that girls had higher prosocial moral orientation than did boys, but there was no significant gender differences in their overall moral judgment [12]. Eisenberg et al. [4] also used their longitudinal findings to show that girls were better than boys in some types of moral reasoning (e.g. other-oriented thinking and prosocial norms).

The effect of economic disadvantage on adolescent moral competence and moral development is unclear as there are limited studies in the field. In terms of family socioeconomic status, Theokas and Lerner [15] showed a positive correlation between household income and positive youth development outcomes based on their study on fifth graders in the US. Hyde et al. [16] also found that the negative early childhood experience (e.g. parental rejection) contributed to subsequent moral disengagement and antisocial behavior among adolescent boys growing up in low-income families. However, few studies had strong evidence to support the negative association of familial economic disadvantage and moral development.

Besides the impact of economic disadvantage on moral competence, the influence of non-intact family (e.g. parental divorce) on adolescent morality was also examined in this study. Some longitudinal research showed that adolescents from divorced/separate families did not differ in their self-reported feeling of shame and guilt (e.g. indicators of morality) when compared to those from intact families [17]. Nevertheless, previous studies on the Project P.A.T.H.S showed that the factors of non-intact family and poor family had negative correlations with adolescent's risk behavior and delinquency $[11,18]$. In short, there is a dearth of research evidence demonstrating that parental marital disruption is harmful to adolescent moral development. Hence, it is worthy to further explore the longitudinal effects of family intactness on adolescent moral competence.

In addition to family economic disadvantage and family intactness, family functioning (e.g. cohesion and communication among family members) positively predicted adolescent external morality, especially in their moral thoughts and moral decision-making [19]. In their longitudinal studies on delinquency trajectories in mid-adolescence, Wiesner and Windle [20] stated that unsupportive family environments (e.g. perceived insufficient emotional and moral support provided by family) predicted the offensive behavior of the adolescents. Although this study did not directly address the impact of family support on adolescent moral competence, it provides research evidence to suggest that family functioning is an important predictor of adolescent moral development.

Besides the aforementioned socio-demographic and family predictors of adolescent moral competence, the impact of the parent-child subsystem on adolescent moral competence is also worth noting [21, 22]. Most studies on the subsystem of parent-child focused on parental warmth, parental monitoring, and parent-child interaction. Smetana et al. [23] reviewed the cross-sectional and longitudinal studies on adolescent development in the family context. They showed that more family interaction resulted in more mature moral reasoning and parents had a long-term impact on children's moral values. Some scholars argued that better parent-child relations and paternal supervision predicted better development of social competence in children [24] as well as their empathy and prosocial behavior [25]. Authoritative parenting (e.g. better support and demands for appropriate behavior and control) was linked to a higher level of moral judgment in adolescence [26].

Given the paucity of empirical studies examining parent-child subsystem quality which contains multiple dimensions of the parent-child relational quality, Shek and Law [22, 27] developed and validated two assessment tools to assess father-child subsystem quality and mother-child subsystem quality (including behavior control, psychological control, and parent-child quality). From the previous studies, father-child subsystem quality and mother-child subsystem quality (except mother's 
behavior control) significantly predicted Internet addiction behavior in adolescents [22]. Paternal and maternal parent-child subsystem quality also had significant correlation with psychosocial wellbeing, substance abuse, and delinquency of adolescents in poor families [28]. Therefore, it was expected these two family variables would have correlation with the adolescent moral competence.

\section{Research questions and hypotheses}

Using six waves of data, we estimated the growth curves of adolescent moral competence and explored whether gender, economic disadvantage, family intactness, family functioning, father-child subsystem quality, and mother-child subsystem quality were related to the initial status as well as patterns of change of moral competence in adolescence. Based on the aforementioned literature review, the main questions and hypotheses were as follows:

a. Does adolescent moral competence change over adolescent years? Based on previous research [4, 5, 12-14, 26], it was predicted that adolescent moral competence would increase throughout the adolescent years (Hypothesis 1).

b. Is gender related to adolescent moral competence? Based on previous research [4, 12], it was predicted that adolescent girls would have higher moral competence than adolescent boys (Hypothesis 2).

c. Is economic disadvantage related to adolescent moral competence? With reference to previous research findings $[15,16]$, our prediction was that poor adolescents would have a lower level of moral competence (Hypothesis 3).

d. Is family intactness related to adolescent moral competence? Following the previous studies [11, 17, 18], we expected that adolescents growing up in non-intact families would have lower moral competence than those growing up in intact families (Hypothesis 4).

e. Is family functioning related to adolescent moral competence? Based on the previous research [19, 20], it was predicted that family functioning would be positively related to adolescent moral competence (Hypothesis 5).

f. Is parent-child subsystem quality related to adolescent moral competence? Based on the previous research [21-23, 26-28], it was predicted that fatherchild subsystem quality and mother-child subsystem quality would be positively related to adolescent moral competence (Hypothesis 6 and Hypothesis 7, respectively).

\section{Methods}

This longitudinal research project was implemented in the 2009/2010 academic year which lasted for 6 years with the collection of six waves of data. More details of this research project can be seen in Shek and Ma's paper [29]. A total of 3328 secondary school students (Grade 7) participated in Wave 1. They were invited to complete the same questionnaire assessing their psychosocial adjustment and family processes in all 6 years. In Wave 6 , the participants studied in Grade 12 (the final year of their secondary school study). The attrition rates of the participants ranged from $12.7 \%$ to $28.3 \%$ from Wave 2 to Wave 6. School, parent and student consent was obtained in prior to the study. All collected questionnaires and data were analyzed by a team of well-trained researchers.

\section{Instruments}

Moral competence refers to how adolescents perceive their competence to judge on what is right or wrong, to conduct moral behavior, and to respect the rules, laws, and social justice $[3,7,8]$. It is one of the 15 positive youth development constructs measured by the Chinese positive youth development scale [8]. Although six questions were intrinsic to the original scale, three questions were used in this study in a six-point Likert-type scale: (1) "I have high moral expectation about my behavior"; (2) "I will fulfill my promise"; (3) "I have the habit of self-evaluation". Moral competence was calculated by the average score of these three items.

Economic disadvantage, family intactness, and family functioning: Economic disadvantage was assessed by one question concerning whether the participant's family had received comprehensive social security assistance (CSSA) in Hong Kong. If the adolescent indicated that his/her family had received CSSA, then he/she was considered as having economic disadvantage (a relatively poor family).

Family intactness refers to the reported parental marital status (1=divorced but not married, $2=$ separated but not remarried, $3=$ first marriage, $4=$ second or subsequent marriage, $5=$ others). Only the participants with parents in their first marriage were considered as having family intactness.

Family functioning was measured by the Chinese family assessment instrument (CFAI) with three subscales: (1) mutuality (mutual support, love, and concerns among family members), (2) communication (frequency and nature of interaction among family member), and (3) conflicts and harmony (the presence of conflicts and harmonious behavior in family). The scale had been developed and validated in previous studies [30, 31]. With scores in the dimension of conflicts and harmony reverse keyed, family functioning was indicated by a mean score of all items, with a higher score indicating a more supportive family environment.

Parental-child subsystem quality: In this study, father-child subsystem quality and mother-child subsystem quality were regarded as two separate measures to assess differences between paternal and maternal parenting in adolescent moral development. These two variables share the same set of questions (totally 17 items, in a five-point Likert-type scale) measuring father and mother separately. Taking father-child subsystem quality as example, the measure includes 
three components: (1) father's behavioral control (seven items), (2) father's psychological control (four items in reversed rating), and (3) the father-child relational quality (six items). The psychometric properties of this scale were validated through exploratory and confirmatory factor analyses [22, 27].

\section{Data analyses}

The data were analyzed by the Statistical Package for the Social Sciences (SPSS) [32]. Descriptive analyses were conducted to calculate the mean scores of moral competence, family functioning, fatherchild subsystem quality, and mother-child subsystem quality in all six waves. Reliabilities of these variables across six waves were also examined. Next, correlations of the Wave 1 socio-demographic variables, family functioning, father-child subsystem quality, and mother-child subsystem quality, and the moral competence (Wave 1 to Wave 6) were examined.

Furthermore, linear mixed models (LMM) were conducted to test and compare the unconditional mean model, unconditional growth model, and conditional growth model, respectively. Unconditional mean model (Model 1) was used to test the within-individual variance without adding predictors. The unconditional growth model (Model 2) was then tested with "Time" as the only level-1 predictor. In the conditional growth models (Model 3 and Model 4), sociodemographic factors were first added as the level-2 predictors in the linear growth curve model of Model 3. In Model 4, family functioning, father-child subsystem quality, and mother-child subsystem quality were then added as the level-2 predictors with the initial status and socio-demographic factors controlled. Based on the results of LMM, the growth trajectory of moral competence was plotted based on Model 2 (see Figure 1). Besides, prototypical plots of moral competence in terms of the above independent variables were also generated based on Model 4 (see Figures 2 and 3).

\section{Results}

In Table 1, results showed that the scales of moral competence, family functioning, father-child subsystem quality, and mother-child subsystem quality were reliable, with

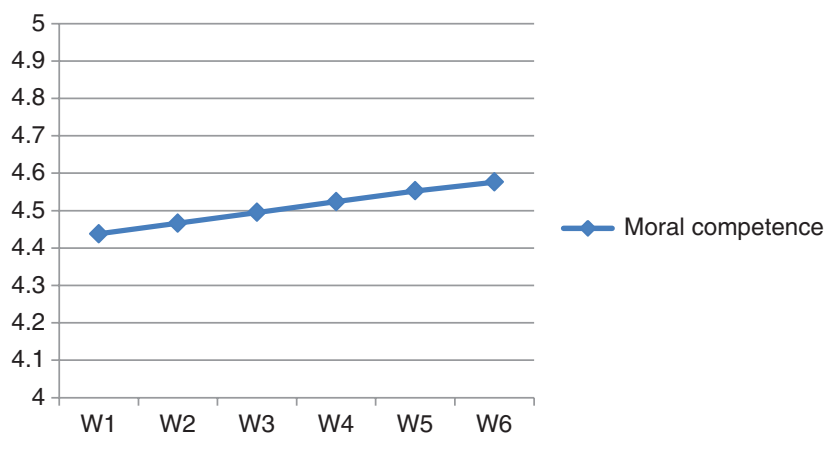

Figure 1: Growth trajectory of the overall sample. The figure was based on Model 2.
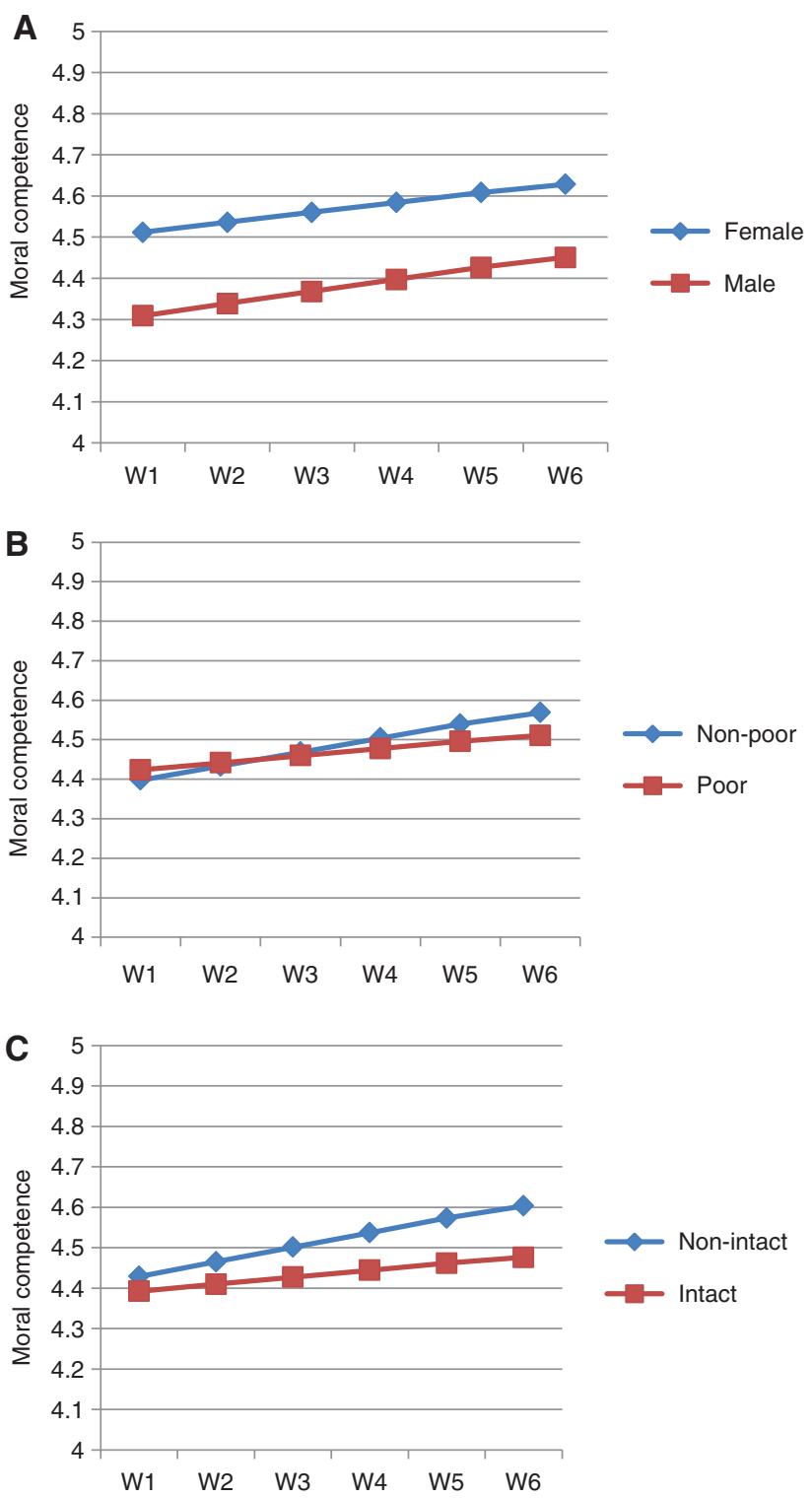

Figure 2: Growth trajectories by socio-demographic variables. (A) Gender, (B) economic disadvantage, and (C) family intactness. The figures were plotted based on Model 4.

the $\alpha$-values above 0.70 in all cases. In Table 2, Pearson's correlation showed that: (1) there were significant correlations of moral competence across six waves; (2) moral competence was negatively related to gender (i.e. female adolescents had higher moral competence than male adolescents) but positively related to family functioning, father-child subsystem quality, and mother-child subsystem quality across six waves (i.e. higher family functioning and parent-child subsystem quality were related to higher level of moral competence). These findings supported the hypotheses posed at the beginning of the study. 
A

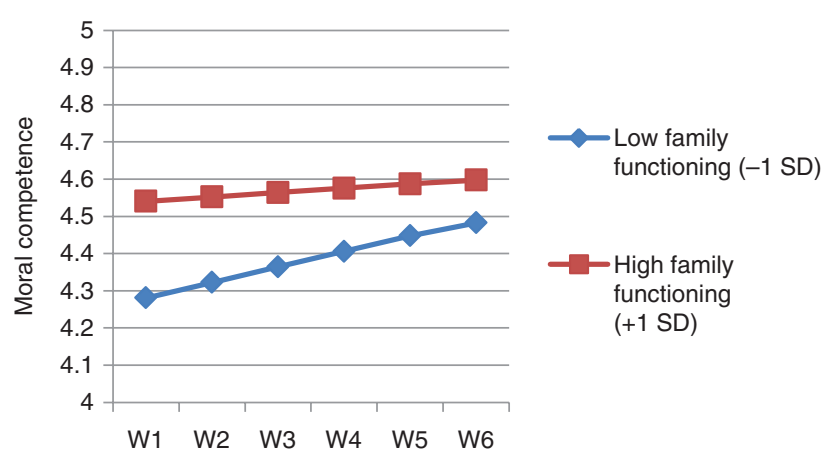

B
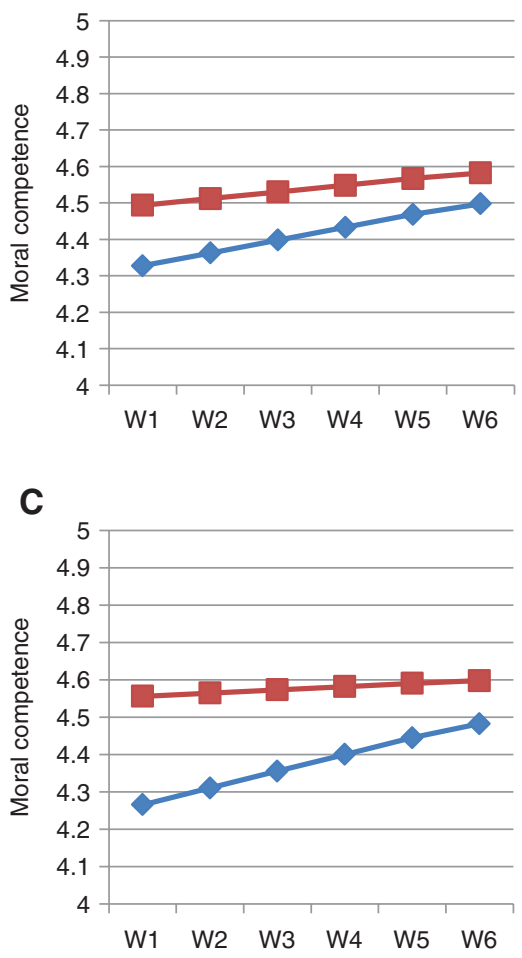

Figure 3: Growth trajectories by family functioning, father-child subsystem quality, and mother-child subsystem quality.

(A) Family functioning, (B) father-child subsystem quality, and

(C) mother-child subsystem quality. The figures were plotted based on Model 4. High level indicates 1 SD higher than the mean value; low level indicates 1 SD lower than the mean value.

To further examine the prediction of different variables on moral competence, LMM analyses were conducted. Firstly, the unconditional mean model (Model 1, in Table 3) was tested to identify the sources of variance of adolescent moral competence using intra-class correlation coefficient (ICC) as an indicator [33]. As calculated, ICC had a value of 0.4752 (47.52\% of total variance of moral competence could be explained by inter-individual variables), which suggested that it was necessary to further examine the relationships between moral competence and different level-2 predictors.

Next, the unconditional growth model (Model 2, in Table 3) was tested which showed a better-fit than the unconditional mean model (Model 1), $\left[\Delta \chi^{2}(3)=396.804\right.$; $\mathrm{p}<0.001 ; \Delta \mathrm{AIC}=390.804 ; \Delta \mathrm{BIC}=367.695]$. The initial level of moral competence was high $\left(\gamma_{00}=4.375, p<0.001\right)$. The random coefficient of time (level-1 predictor) in the linear slope $\left(\gamma_{10}=0.029, \mathrm{p}<0.001\right)$ showed there was a 0.029 mean unit of increase of moral competence in each year. Based on Model 2, the growth trajectory of moral competence was plotted, and it also showed an increase trend in all 6 years (Figure 1). Therefore, Hypothesis 1 was supported. In terms of random effect, $14.3 \%$ between-individual variance of moral competence could be explained by time effect (level-1 predictor). In addition, the between-subject variances were both significant (intercept $u_{0 j}=0.459$, $\mathrm{p}<0.001$; slope $u_{1 j}=0.013, \mathrm{p}<0.001$ ), which indicated the possibility of higher-level inter-personal predictors in explaining the variances in intercept and linear growth curve.

Finally, the conditional growth models (Model 3 and Model 4, in Table 4) were examined. In Model 3, three socio-demographic variables (gender, economic disadvantage, family intactness) were first tested as the level-2 predictors in the linear growth curves of moral competence over six waves. Model 3 had a model fitted better than Model 2 [ $\Delta \chi^{2}(6)=5401.131 ; \mathrm{p}<0.001 ; \Delta \mathrm{AIC}=5397.131$; $\Delta \mathrm{BIC}=5383.710]$. Then, in Model 4, family functioning, father-child subsystem quality, and mother-child subsystem quality were added as the level-2 predictors with the initial status and socio-demographic predictors controlled. Results in Model 4 showed a better fit than Model $3\left[\Delta \chi^{2}(6)=6837.355 ; \mathrm{p}<0.001 ; \quad \Delta \mathrm{AIC}=6825.355\right.$; $\Delta \mathrm{BIC}=6784.378]$. Therefore, Model 4 was finally selected as the model to explain the level-2 predictors on initial status and rate of change in moral competence.

In Model 4, the random coefficient of gender was significant in initial status $\left(\gamma_{01}=-0.101, \mathrm{p}<0.001\right)$ but not in the linear slope $\left(\gamma_{11}=0.003\right)$, which meant that female adolescents had a higher level of moral competence than male adolescents in Wave 1, but female adolescents did not have a significantly faster growth of moral competence than male adolescents. Therefore, Hypothesis 2 was supported. Another two socio-demographic variables (economic disadvantage and family intactness) were not significantly associated with the initial status and rate of change of moral competence. In addition, comparison of the linear growth curves of economic disadvantage (poor versus non-poor students) and family intactness (intact 


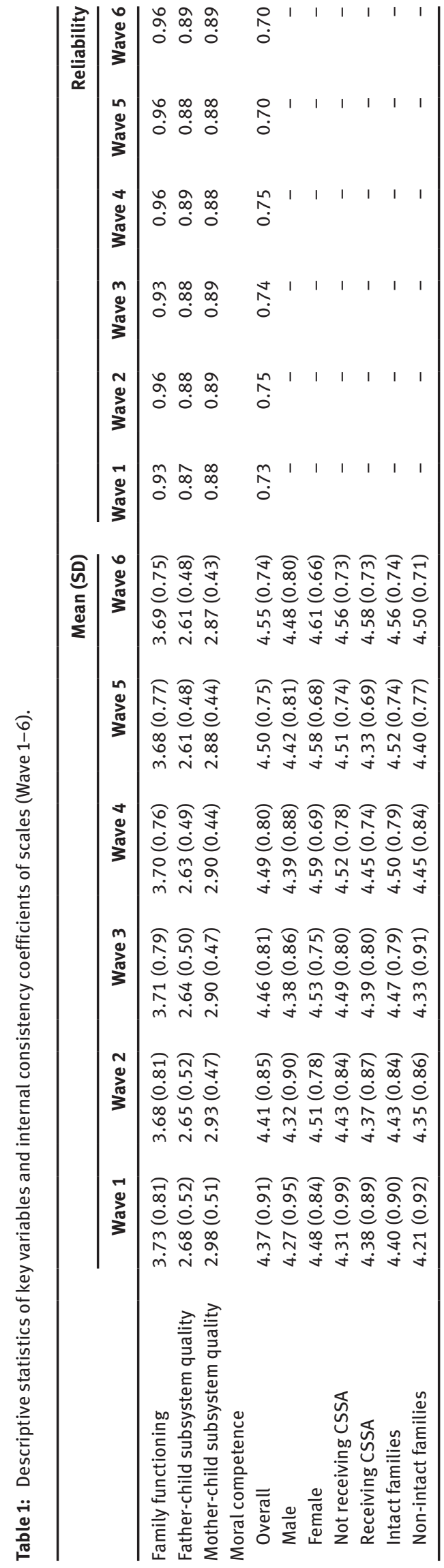

versus non-intact students), there were merely minor differences between them (see Figure 2). Therefore, Hypothesis 3 and 4 were not supported.

Family functioning was positively associated with the initial status of moral competence $\left(\gamma_{06}=0.130, \mathrm{p}<0.001\right)$ but negatively related with the rate of change $\left(\gamma_{16}=-0.015\right.$, $\mathrm{p}<0.05)$. The findings suggested that adolescents from families with better family functioning had a high initial level of moral competence but slower growth rate than that of who came from families with relatively poorer family functioning. Father-child subsystem quality was positively associated with the initial status of moral competence $\left(\gamma_{04}=0.083, p<0.001\right)$. It showed adolescents with higher perceived paternal parenting quality had a higher initial level of moral competence than did those with lower quality of paternal parenting. Mother-child subsystem quality was positively associated with the initial status of moral competence $\left(\gamma_{05}=0.145, \mathrm{p}<0.001\right)$ but negatively related to the rate of change $\left(\gamma_{15}=-0.018, \mathrm{p}<0.001\right)$. The findings suggested that adolescents with higher perceived parenting quality of the mothers had a higher initial level of moral competence but slower growth rate than those with lower one. In terms of the random effects, $25.1 \%$ of the total between-individual variance in the intercept could be explained by the level- 2 predictors, and $15.4 \%$ of the total variance in growth rate can be explained by the level-2 predictors.

The above results on the predictors in Model 4 were further elaborated by the prototypical plots (in Figure 3). In Figure 3, adolescents with higher family functioning, father-child subsystem quality, and mother-child subsystem quality had higher mean scores than did those with relatively lower family functioning, father-child subsystem quality, and mother-child subsystem quality across six waves. Adolescents with better perceived family functioning and higher father-child as well as mother-child subsystem quality were stable in the development of moral competence comparing to the ones who had relatively poorer family functioning and lower father-child/ mother-child subsystem quality. Together with the findings based on Pearson's correlation analyses (in Table 2), family functioning and parent-child relational qualities could positively predict the initial level of adolescent moral competence, which provided support for Hypotheses 5, 6 and 7.

\section{Discussion}

A review of the literature shows that there are few longitudinal studies examining the development of moral 
Table 2: Correlations among variables.

\begin{tabular}{|c|c|c|c|c|c|c|}
\hline Variables & MC & SMC & TMC & FMC & GMC & QMC \\
\hline MC & 1 & & & & & \\
\hline SMC & $0.487^{\mathrm{g}}$ & 1 & & & & \\
\hline TMC & $0.419^{g}$ & $0.533^{g}$ & 1 & & & \\
\hline FMC & $0.391^{\mathrm{g}}$ & $0.476^{\mathrm{g}}$ & $0.533^{\mathrm{g}}$ & 1 & & \\
\hline GMC & $0.370^{g}$ & $0.432^{\mathrm{g}}$ & $0.474^{\mathrm{g}}$ & $0.554^{\mathrm{g}}$ & 1 & \\
\hline QMC & $0.295^{\mathrm{g}}$ & $0.382^{\mathrm{g}}$ & $0.446^{\mathrm{g}}$ & $0.479^{g}$ & $0.530^{\mathrm{g}}$ & 1 \\
\hline Gender $^{\mathrm{a}}$ & $-0.117^{g}$ & $-0.114^{\mathrm{g}}$ & $-0.087^{g}$ & $-0.130^{\mathrm{g}}$ & $-0.107^{g}$ & $-0.086^{\mathrm{g}}$ \\
\hline Economic disadvantage $^{\mathrm{b}}$ & -0.023 & -0.025 & -0.034 & -0.033 & $-0.043^{h}$ & -0.026 \\
\hline Family intactness ${ }^{c}$ & $0.079^{\mathrm{g}}$ & $0.044^{\mathrm{h}}$ & 0.037 & 0.018 & $0.041^{\mathrm{h}}$ & 0.024 \\
\hline Father-child subsystem quality ${ }^{d}$ & $0.321^{\mathrm{g}}$ & $0.215^{\mathrm{g}}$ & $0.186^{\mathrm{g}}$ & $0.198^{g}$ & $0.198^{\mathrm{g}}$ & $0.143^{g}$ \\
\hline Mother-child subsystem ${ }^{\mathrm{e}}$ quality & $0.350^{\mathrm{g}}$ & $0.281^{\mathrm{g}}$ & $0.230^{\mathrm{g}}$ & $0.187^{g}$ & $0.211^{\mathrm{g}}$ & $0.158^{g}$ \\
\hline Family functioning ${ }^{f}$ & $0.353^{\mathrm{g}}$ & $0.272^{\mathrm{g}}$ & $0.224^{\mathrm{g}}$ & $0.195^{g}$ & $0.207^{g}$ & $0.163^{\mathrm{g}}$ \\
\hline
\end{tabular}

MC, Moral competence at Wave 1; SMC, moral competence at Wave 2; TMC, moral competence at Wave 3; FMC, moral competence at Wave

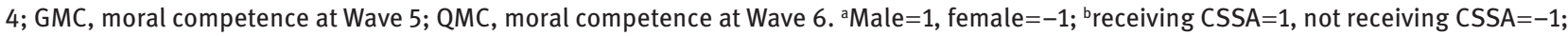

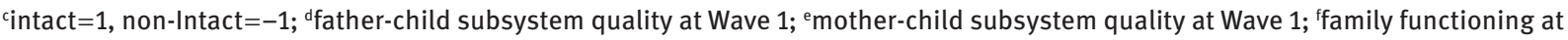
Wave $1 ;{ }^{\mathrm{g} p}<0.01 ;{ }^{\mathrm{h}} \mathrm{p}<0.05$.

Table 3: Results of LMM models with level-1 predictor.

\begin{tabular}{|c|c|c|c|c|c|}
\hline & & \multicolumn{2}{|c|}{ Model 1} & \multicolumn{2}{|c|}{ Model 2} \\
\hline & & Estimate & SE & Estimate & SE \\
\hline \multicolumn{6}{|l|}{ Fixed effects } \\
\hline Intercept & $\beta_{0 j}$ & & & & \\
\hline Intercept & $\gamma_{00}$ & $4.435^{\mathrm{a}}$ & 0.011 & $4.375^{a}$ & 0.014 \\
\hline Linear slope & $\beta_{1 j}$ & & & & \\
\hline Time & $\gamma_{10}$ & & & $0.029^{a}$ & 0.003 \\
\hline \multicolumn{6}{|c|}{ Random effects } \\
\hline \multicolumn{6}{|c|}{ Level-1 (within) } \\
\hline Residual & $r_{i j}$ & $0.360^{\mathrm{a}}$ & 0.004 & $0.317^{a}$ & 0.004 \\
\hline \multicolumn{6}{|c|}{ Level-2 (between) } \\
\hline Intercept & $u_{0 j}$ & $0.326^{\mathrm{a}}$ & 0.010 & $0.459^{\mathrm{a}}$ & 0.016 \\
\hline Time & $u_{1 j}$ & & & $0.013^{\mathrm{a}}$ & 0.001 \\
\hline \multicolumn{6}{|l|}{ Fit statistics } \\
\hline Deviance & & 35218.865 & & 34822.061 & \\
\hline AIC & & 35224.865 & & 34834.061 & \\
\hline $\mathrm{BIC}$ & & 35247.975 & & 34880.280 & \\
\hline $\mathrm{df}$ & & 3 & & 6 & \\
\hline
\end{tabular}

Model 1, Unconditional mean model; Model 2, unconditional growth model. ${ }^{\mathrm{a}} \mathrm{p}<0.001,{ }^{\mathrm{b}} \mathrm{p}<0.01$.

competence in Chinese adolescents. There are several unique characteristics of the study. First, data were collected over 6 years to understand adolescent moral competence. In fact, this is the first known scientific study collecting six waves of data in different Chinese contexts. Second, a large sample of Chinese students was randomly selected to enhance the generalizability of the findings. Third, validated instruments were used in the study. Fourth, individual growth curve models were constructed and tested in the study. Finally, a wide range of developmental outcome indicators were included in the study.

This study provides a rich picture on the normative profile of adolescent moral competence across time in Hong Kong. It shows that adolescent moral competence was related to gender, family functioning as well as parent-child relational quality. Several interesting observations can be highlighted from the present study. First, gender was significantly related to initial status of moral competence, with female adolescents showing a higher initial level of moral competence than male adolescents. However, gender differences were not found in the developmental trajectories. Second, good perceived family functioning and quality of parental/maternal parenting showed a significant impact on the growth trajectory of adolescent moral competence across time. These findings are generally consistent with the findings in the scientific literature.

The present findings showed that mother-child subsystem quality had a significant effect on the growth trajectory of moral competence in adolescence. It echoes the previous findings reported by Shek et al. [18, 22] that adolescents had a closer relationship with mother, although mother had more behavioral and psychological control on their children. On the other hand, fatherchild subsystem quality could be considered as equally or even more influential than mother-child subsystem quality in adolescent's healthy development because of the patriarchal power of fathers (e.g. control family resource, decision-making) in the Chinese culture [22, $34,35]$. Although the growth rates of moral competence in adolescents with lower parent-child relational quality were faster than those experienced higher parent-child 
Table 4: Results of LMM models with level-2 predictors.

\begin{tabular}{|c|c|c|c|c|c|}
\hline & & \multicolumn{2}{|c|}{ Model 3} & \multicolumn{2}{|c|}{ Model 4} \\
\hline & & Estimate & SE & Estimate & SE \\
\hline \multicolumn{6}{|l|}{ Fixed effects } \\
\hline Intercept & $\beta_{0 j}$ & & & & \\
\hline Intercept & $\gamma_{00}$ & $4.329^{d}$ & 0.029 & $4.41^{d}$ & 0.032 \\
\hline Gender $^{\mathrm{a}}$ & $\gamma_{01}$ & $-0.107^{d}$ & 0.015 & $-0.101^{d}$ & 0.016 \\
\hline Economic disadvantage $^{b}$ & $\gamma_{02}$ & -0.008 & 0.030 & 0.013 & 0.033 \\
\hline Family intactness ${ }^{c}$ & $\gamma_{03}$ & $0.069^{\mathrm{e}}$ & 0.023 & -0.018 & 0.025 \\
\hline Father-child subsystem quality & $\gamma_{04}$ & & & $0.083^{d}$ & 0.020 \\
\hline Mother-child subsystem quality & $\gamma_{05}$ & & & $0.145^{d}$ & 0.020 \\
\hline Family functioning & $\gamma_{06}$ & & & $0.130^{\mathrm{d}}$ & 0.022 \\
\hline Linear slope & $\beta_{1 j}$ & & & & \\
\hline Intercept & $\gamma_{10}$ & $0.032^{\mathrm{d}}$ & 0.007 & $0.027^{e}$ & 0.009 \\
\hline Gender $^{\mathrm{a}}$ & $\gamma_{11}$ & 0.005 & 0.004 & 0.003 & 0.004 \\
\hline Economic disadvantage $^{\mathrm{b}}$ & $\gamma_{12}$ & -0.007 & 0.008 & -0.009 & 0.009 \\
\hline Family intactness ${ }^{c}$ & $\gamma_{13}$ & $-0.015^{f}$ & 0.006 & -0.009 & 0.006 \\
\hline Father-child subsystem quality & $\gamma_{14}$ & & & -0.008 & 0.005 \\
\hline Mother-child subsystem quality & $\gamma_{15}$ & & & $-0.018^{d}$ & 0.005 \\
\hline Family functioning & $\gamma_{16}$ & & & $-0.015^{f}$ & 0.006 \\
\hline \multicolumn{6}{|l|}{ Random effects } \\
\hline \multicolumn{6}{|l|}{ Level-1 (within) } \\
\hline Residual & $r_{i j}$ & $0.314^{d}$ & 0.005 & $0.305^{d}$ & 0.005 \\
\hline \multicolumn{6}{|l|}{ Level-2 (between) } \\
\hline Intercept & $u_{0 j}$ & $0.443^{d}$ & 0.017 & $0.344^{\mathrm{d}}$ & 0.016 \\
\hline Time & $u_{1 j}$ & $0.013^{d}$ & 0.001 & $0.011^{\mathrm{d}}$ & 0.001 \\
\hline \multicolumn{6}{|l|}{ Fit statistics } \\
\hline Deviance & & 29267.449 & & 22430.094 & \\
\hline AIC & & 29291.449 & & 22466.094 & \\
\hline $\mathrm{BIC}$ & & 29381.902 & & 22597.524 & \\
\hline df & & 12 & & 18 & \\
\hline
\end{tabular}

Model 3, conditional growth curve model (only with socio-demographic variables); Model 4, conditional growth curve model (adding family correlates). ${ }^{a}$ Male $=1$, female $=-1$; ${ }^{b}$ receiving $C S S A=1$, not receiving CSSA $=-1 ;$ cintact $=1$, non-intact $=-1 ;{ }^{d} p<0.001 ;{ }^{e} p<0.01 ;{ }^{f} p<0.05$.

relational quality, the absolute level of moral competence was still higher in those with higher parent-child relational quality. The findings of the study provide good support for this claim that family plays an important role in the development of moral competence in Chinese adolescents.

The present findings did not provide support for the relationship between demographic family variables (family intactness and family socio-economic status) and adolescent moral competence. There are two possible explanations for this observation. First, although parental divorce could be detrimental to children's healthy development, such negative outcomes may be offset by the effects such as good child-rearing quality of divorced parents and adolescents' own coping with divorce and their social competence [36]. As such, future studies should be conducted to examine such possibilities. Second, as societal acceptance of poverty and non-intact families has become greater, the detrimental effect of such family structural characteristics on adolescent development may become less. In future studies, it is suggested that family processes in families experiencing economic disadvantage and nonintactness should be investigated to further understand how family process moderates the effects of economic disadvantage and family intactness on adolescent development of moral competence.

Although the present study is pioneer in nature, it has several limitations. First, as only adolescent-report of moral competence was assessed, it should be helpful if other measures using reports of significant others are included. Second, as only a few items were used to measure moral competence, more items and more dimensions of the concept should be added in future. Third, future studies may consider adding other social and ecological predictors in the model, such as one's religious beliefs, identification with community norms, peer influence [26], role modeling of siblings [14], as well as school education and social media. In addition, father as a role 
model of morality and the role of father-child interaction in adolescent healthy development should also be further explored. Despite the limitations, the findings of this study suggests that more policy and social service initiatives should be considered to promote adolescent moral development. For example, in the Project P.A.T.H.S. [37, 38], positive youth development programs have been shown to promote development in adolescents, including moral competence [39].

Acknowledgments: The paper and the Project P.A.T.H.S. were financially supported by the Hong Kong Jockey Club Charities Trust.

\section{References}

1. Catalano RF, Berglund ML, Ryan JA, Lonczak HS, Hawkins JD. Positive youth development in the United States: research findings on evaluations of positive youth development programs. Anna Am Acad Polit Soc Sci 2004;591:98-124.

2. Park N, Peterson C. Moral competence and character strengths among adolescents: the development and validation of the values in action inventory of strengths for youth. J Adolescence 2006;29:891-909.

3. Ma HK. Moral competence as a positive youth development construct: a conceptual review. ScientificWorldJ 2012. doi:10.1100/2012/590163.

4. Eisenberg N, Cumberland A, Guthrie IK, Murphy BC, Shepard SA. Age changes in prosocial responding and moral reasoning in adolescence and early adulthood. J Res Adolescence 2005;15:235-60.

5. Killen M, Smetana J, editors. Handbook of moral development. Mahwah, NJ: Lawrence Erlbaum, 2010.

6. Lewin-Bizan S, Bowers EP, Lerner RM. One good thing leads to another: cascades of positive youth development among American adolescents. Dev Psychopathol 2010;22:759-70.

7. Ma HK. Moral competence as a positive youth development construct: conceptual bases and implications for curriculum development. Int J Adolesc Med Health 2006;18:371-8.

8. Shek DT, Siu AM, Lee TY. The Chinese Positive Youth Development Scale: a validation study. Res Social Work Pract 2007;17:380-91.

9. Shek DT, Ma CM. Dimensionality of the Chinese positive youth development scale: confirmatory factor analyses. Soc Indic Res 2010;98:41-59.

10. Shek DT, Ma CM. Impact of the Project P.A.T.H.S. on adolescent developmental outcomes in Hong Kong: Findings based on seven waves of data. Int J Adolesc Med Health 2012;24:231-44.

11. Shek DT, Yu L. Longitudinal impact of the project PATHS on adolescent risk behavior: what happened after five years? ScientificWorldJ 2012;2012:316029.

12. Ma HK. The relation of moral orientation and moral judgment to prosocial and antisocial behaviour of Chinese adolescents. Int J Psychol 2003;38:101-11.
13. Fang F, Fang G, Keller M, Edelstein W, Shuster P. A cross cultural study on the development of moral reasoning in friendship in Western and Eastern children and adolescents. Acta Psychol Sinica 2002;34:67-73.

14. Fang G, Fang F, Keller M, Edelstein W, Kehle TJ, Bray MA. Social moral reasoning in Chinese children: a developmental study. Psychol Sch 2003;40:125-38.

15. Theokas C, Lerner RM. Observed ecological assets in families, schools, and neighborhoods: conceptualization, measurement, and relations with positive and negative developmental outcomes. Appl Dev Sci 2006;10:61-74.

16. Hyde LW, Shaw DS, Moilanen KL. Developmental precursors of moral disengagement and the role of moral disengagement in the development of antisocial behavior. J Abnorm Child Psychol 2010;38:197-209.

17. Walter JL, Burnaford SM. Developmental changes in adolescents' guilt and shame: the role of family climate and gender. North Am J Psychol 2006;8:321-38.

18. Shek DT, Lin L. Delinquent behavior in high school students in Hong Kong: sociodemographic, personal, and family determinants. J Pediatr Adolesc Gynecol 2006;29:61-71.

19. White FA, Matawie KM. Parental morality and family processes as predictors of adolescent morality. J Child Fam Stud 2004;13:219-33.

20. Wiesner M, Windle M. Assessing covariates of adolescent delinquency trajectories: a latent growth mixture modeling approach. J Youth Adolescence 2004;33:431-42.

21. Cabrera NJ, Tamis-LeMonda CS, editors. Handbook of father involvement: multidisciplinary perspectives. New York, NY: Routledge, 2013.

22. Shek DT, Law YM. Assessment of parent-child subsystem qualities in Chinese adolescents: behavioral control, psychological control and parent-child relational qualities. Int J Child Health Hum Dev 2015;8:207-17.

23. Smetana JG, Campione-Barr N, Metzger A. Adolescent development in interpersonal and societal contexts. Annu Rev Psychol 2006;57:255-84.

24. McDowell DJ, Parke RD. Parental correlates of children's peer relations: an empirical test of a tripartite model. Dev Psychol 2009;45:224-35.

25. Yoo H, Feng X, Day RD. Adolescents' empathy and prosocial behavior in the family context: a longitudinal study. J Youth Adolescence 2013;42:1858-72.

26. Eisenberg N, Morris A, McDaniel B, Spinrad T. Moral cognitions and prosocial responding in adolescence. In: Lerner RM, Steinberg L, editors. Handbook of adolescent psychology. Volume 1: Individual bases of adolescent development, 3rd ed. Hoboken, NJ: John Wiley, 2009:229-65.

27. Shek DT, Law MY. Parental behavioral control, parental psychological control and parent-child relational qualities: relationships to Chinese adolescent risk behavior. In: Shek DT, Sun RCF, $\mathrm{Ma} \mathrm{CM}$, editors. Chinese adolescents in Hong Kong. Singapore: Springer, 2014:51-69.

28. Shek DT. Paternal and maternal influences on the psychological well-being, substance abuse, and delinquency of Chinese adolescents experiencing economic disadvantage. J Clin Psychol 2005;61:219-34.

29. Shek DT, Ma CM. A longitudinal study of the personal well-being and family quality of life among Chinese adolescents in Hong 
Kong. In: Shek DT, Sun RC, Ma CM, editors. Chinese adolescents in Hong Kong. Singapore: Springer, 2014:19-34.

30. Shek DT. Assessment of family functioning in Chinese adolescents: the Chinese version of the Family Assessment Device. Res Social Work Pract 2002;12:502-24.

31. Shek DT, Ma CM. The Chinese Family Assessment Instrument (C-FAl): hierarchical confirmatory factor analyses and factorial invariance. Res Social Work Pract 2010;20:112-23.

32. IBM SPSS Statistics: Statistical Package for the Social Sciences (SPSS) for Windows (Version 22.0). Somers, NY: IBM Corporation, 2014.

33. Shek DT, Ma CM. Longitudinal data analyses using linear mixed models in SPSS: concepts, procedures and illustrations. ScientificWorldJ 2011;11:42-76.

34. Shek DT. Parenting characteristics and adolescent psychological well-being: a longitudinal study in a Chinese context. Genetic Soc General Psycho Mono 1999;125: 27-44.
35. Shek DT. Paternal and maternal influences on the psychological well-being of Chinese adolescents. Genetic Soc General Psycho Mono 1999;125:269-96.

36. Peterson GW. Family influences on adolescent development. In: Gullotta T, Adams G, editors. Handbook of adolescent behavioral problems. New York, NY: Springer, 2005;27-55.

37. Shek DT, Sun CF. The Project P.A.T.H.S. in Hong Kong: development, training, implementation, and evaluation. J Pediatr Adolesc Gynecol 2013;26:S2.

38. Shek DT, Sun CF, editors. Development and evaluation of positive adolescent training through holistic social programs (P.A.T.H.S.). Heidelberg: Springer, 2013.

39. Shek DT, Ma CM. Impact of the Project P.A.T.H.S. in the junior secondary school years: objective outcome evaluation based on eight waves of longitudinal data. ScientificWorldJ 2012. doi:10.1100/2012/170345. 
Reproduced with permission of the copyright owner. Further reproduction prohibited without permission. 